\title{
Extinction of enhanced latent inhibition
}

\author{
PHIL REED, PANY PETROCHILOS, NATASHA UPAL, and MARTIN BAUM \\ University College London, London, England
}

\begin{abstract}
Hungry rats were used in a classical conditioning procedure in which visual stimuli were paired with food. Conditions in which nonreinforced exposure to a nontarget stimulus was followed by exposure to a simultaneous compound nontarget/target stimulus (a blocking procedure) resulted in enhanced latent inhibition to the target relative to exposure to the nontarget, followed by exposure to the target stimulus alone. A third phase of nonreinforced exposure training, in which the target was exposed alone following the compound, reduced levels of latent inhibition relative to results obtained with the blocking procedure. Experiments also suggested that this was not the result of restoration of associability by the omission of an expected presentation of the nontarget stimulus in the final preexposure phase. These results suggest that enhanced latent inhibition is due to summation of a direct-target-noevent association and a second-order association of these elements via target-nontarget and nontarget-no-event association. Exposure to the target after compound exposure extinguished the target-nontarget association and reduced the sources of no-event learning for the target.
\end{abstract}

Exposure to a stimulus in the absence of reinforcement retards the subsequent emergence of responding to that stimulus during conditioning. A number of theories have been put forward to account for this latent inhibition effect (e.g., Hall \& Honey, 1989; Hall, Kaye, \& Pearce, 1985; Wagner, 1981). The present report focuses specifically on the evidence for the associative interference account of latent inhibition, which suggests that latent inhibition occurs because subjects acquire a conditioned stimulus (CS)-no-event association during preexposure that interferes with subsequent learning of a stimulusevent relationship.

Some have suggested that if the rules governing the formation of a CS-no-event association are equivalent to those governing CS-event associations, then, prior to nonreinforced exposure to a target/nontarget compound, nonreinforced exposure to a nontarget stimulus will establish a nontarget-no-event association that should block acquisition of a target no-event association (Rudy, Krauter, \& Gaffuri, 1976). The target CS, now free from interference, should readily enter into an association with the reinforcer and conditioning should proceed normally. Studies employing such a "blocking-of-latent-inhibition" procedure, however, have generally failed to provide evidence that this treatment leads to greater conditioning than that found in an overshadowing control group that receives only compound exposure prior to conditioning. For example, Honey and Hall (1988) found that presentation of the nontarget stimulus prior to exposure to the nontarget/target compound led to conditioning that was

These data were presented at the Experimental Analysis of Behaviour Group Meeting, London, 1996. Thanks are extended to Geof Hall, Tony Dickinson, Celia Heyes, and Chris Mitchell for helpful criticism of this research. Requests for reprints should be sent to P. Reed, Department of Psychology, University College London, Gower Street, London, WC1E6BT, England (e-mail: p.reed@ucl.ac.uk). slower than that produced by compound preexposure alone. In fact, latent inhibition accrued to the target stimulus to the same extent as it did in a condition in which only the target stimulus was exposed. Furthermore, Reed (1995a, 1995b) noted that, with greater initial nontarget exposure, a blocking treatment resulted not only in retarded conditioning, as compared with compound exposure, but also in enhanced levels of latent inhibition relative to a group first exposed to the nontarget stimulus and then to the target stimulus.

Such failures to note blocking of latent inhibition have lead a number of researchers to abandon the associative interference view of latent inhibition and to develop alternative accounts of the effects of stimulus preexposure (e.g., Honey \& Hall, 1988). However, Reed (1995b) suggested that, somewhat paradoxically, these results actually might be taken as support for the associative interference account.

The blocking procedure is potentially also a higher order conditioning procedure that should allow the formation of associations between the elements of the compound stimulus. Indeed, associations between the elements of a compound stimulus used in a blocking design have been noted in several experiments (e.g., Rescorla \& Colwill, 1983; Speers, Gillan, \& Rescorla, 1980). If latent inhibition is a form of conditioning (and a CS-no-event association is learned during exposure), then some higher order conditioning of latent inhibition to the target stimulus might be expected by virtue of the pairing of the target and nontarget stimuli during compound exposure (a nontarget-no-event association having been established in the first phase of the procedure). A problem for this account is to specify why such higher order conditioning dominates any blocking effect in latent inhibition procedures, whereas blocking is observed in standard conditioning procedures. One possibility stems from observations made by Holland (1980). In that report it 
was noted that the presentation of a US interferes with higher order conditioning in the standard procedure; in the latent inhibition case, of course, no explicit US is presented, so that higher order effects may predominate. Thus, there would be two sources of a target-no-event association in the blocking-of-latent-inhibition design: one acquired directly through presentation of the target stimulus in the absence of a reinforcer, and the other through an association of the target with the nontarget stimulus and, hence, to the no event representation associated with the nontarget. The summation of these two associations between target and no event may lead to greater retardation of conditioning (i.e., enhanced latent inhibition) relative to that of a group in which the targetno-event association is acquired only via first-order conditioning. Thus, the enhanced latent inhibition observed by Reed (1995a, 1995b) could be accommodated by an associative interference account of latent inhibition.

The present series of experiments was designed to explore further the implications of the preceding associative analysis. Specifically, it is suggested that if enhanced latent inhibition is the result of a summation of the two sources of target-no-event learning stated above, then exposure to the target alone, following the compoundexposure phase of the blocking-of-latent-inhibition procedure might extinguish the target-nontarget association. This would abolish one source of target- no-event learning, and conditioning following this "extinction" treatment might be better than that following the blocking-oflatent-inhibition treatment. After the latter procedure there would still be two sources for the target-no-event association. This result would be counterintuitive, since conditioning would be better following greater amounts of nonreinforced exposure to the target, and also would lend support to the view that associative interference could be responsible, at least in part, for producing latent inhibition.

\section{EXPERIMENT 1}

In the first experiment, we sought to replicate the finding that a blocking-of-latent-inhibition procedure (exposure to a simultaneous compound of two visual stimuli following exposure to the nontarget stimulus) produces greater levels of latent inhibition than does preexposure to the target alone following preexposure to a nontarget stimulus (Reed, 1995a, 1995b). In addition, we sought to demonstrate that a third phase of preexposure, in which the target stimulus was presented alone after compound exposure, would lead to conditioning to the target that was more rapid than that noted in the group receiving the blocking-of-latent-inhibition procedure.

\section{Method}

Subjects. Thirty-two experimentally naive, male, SpragueDawley rats were used. The subjects were all $3-4$ months old at the start of the experiment, had a free-feeding body weight range of $305-355 \mathrm{~g}$, and were maintained at $85 \%$ of this weight throughout the experiment. The rats were housed in groups of 4 and had constant access to water in the home cage.
Apparatus. Training was conducted in four identical operantconditioning chambers (Campden Instruments Ltd.) from which the levers had been withdrawn. The chambers were ventilated by a fan that also provided a $65-\mathrm{dB}(\mathrm{A})$ background masking noise. Reinforcement (a 45-mg food pellet) could be delivered to a food tray, which was covered by a clear Perspex, hinged flap. A microswitch was operated when the flap was opened, and closures of the microswitch were recorded as single responses. A bulb, located so as to illuminate the food tray, could be used to provide one of the stimuli. This stimulus was the target stimulus for all experiments in the present report. One jewelled light (the overhead light) was located in the center of the chamber ceiling, and another was located on the wall above the magazine tray (the center light). These lights were used as nontarget stimuli. Other than these visual stimuli, the chamber was not illuminated during the course of the experiment.

Procedure. The subjects were divided into four groups $(n=8)$ matched in terms of free-feeding body weights. In Phase 1 , three groups of subjects (Groups $\mathrm{A} / \mathrm{AB}, \mathrm{A} / \mathrm{B}$, and $\mathrm{A} / \mathrm{AB} / \mathrm{B}$ ) received twelve 30 -min sessions in which they were presented with ten 30 sec presentations of the nontarget (A) stimulus (the overhead light). The rats in Group Cond were placed in the chamber for twelve 30 min sessions, during which they did not receive stimulus presentations. In Phase 2, Groups $\mathrm{A} / \mathrm{AB}$ and $\mathrm{A} / \mathrm{AB} / \mathrm{B}$ received four 30 -min sessions in which they were presented with ten $30-\mathrm{sec}$ compound presentations of the overhead light and traylight (AB). Group A/B received four 30 -min sessions in which they were presented with ten 30 -sec presentations of the traylight (the target). Group Cond was placed in the chambers for four 30-min sessions, during which they were not presented with stimuli. In Phase 3 , Group A/AB/B received four 30-min sessions in which they were presented with ten $30-\mathrm{sec}$ presentations of the traylight (B). The remaining three groups were placed in the chamber for four 30 -min sessions but did not receive programmed stimulus presentations.

In the final, conditioning, phase, all groups received four $30-\mathrm{min}$ sessions in which 30 -sec presentations of the traylight were followed by the presentation of one food pellet. All interstimulus intervals (ISIs) in the present experiment were $2.5 \mathrm{~min}$.

\section{Results and Discussion}

Figure 1 displays the group mean elevation ratios for each five-trial block of the conditioning phase of the study. The elevation ratio for a trial was calculated by counting the number of magazine entries made during the CS, and dividing this number by the magazine entries made during the CS and the 30 -sec stimulus-free period immediately prior to the CS. Inspection of Figure 1 reveals that the groups acquired the magazine entry response in the presence of the target stimulus at different rates. Group Cond came to have a higher elevation ratio than any of the other groups. Group A/AB displayed very little evidence of the acquisition of conditioned responding, and had a lower elevation ratio than either Group A/B or Group A/AB/B. By the end of training, the latter two groups had elevation ratios similar to each other.

A two-factor analysis of variance (ANOVA) with group and block as factors revealed no main effect of group $(p>.10)$, a significant effect of block $[F(7,196)=$ $15.09, p<.001]$, and a significant interaction between these factors $[F(21,196)=2.55, p<.001]$. To analyze these data further, the simple main effect of group on each block was calculated. This revealed a significant group difference on Blocks 6,7 , and 8 [smallest $F(3,159)=3.18$, $p<.01]$. Newman-Keuls tests were conducted on the 


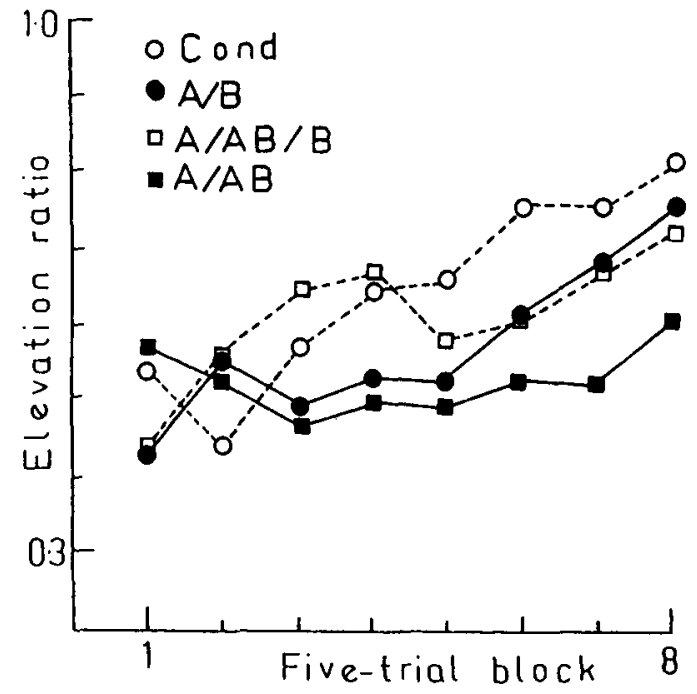

Figure 1. Results from Experiment 1. Group-mean elevation ratios for each five-trial block of conditioning with stimulus $B$. Group $A / A B=$ exposure to nontarget $(A)$ before target/nontarget compound ( $A B)$. Group $A / B=$ exposure to nontarget $(A)$ prior to target (B). Group $A / A B / B=$ exposure to nontarget $(A)$, then target/nontarget compound (AB). Group Cond $=$ no preexposure.

blocks for which a significant effect of group was demonstrated. On Block 6, Group Cond differed from each of the other groups. On Block 7, Group Cond differed from Groups $\mathrm{A} / \mathrm{AB} / \mathrm{B}$ and $\mathrm{A} / \mathrm{AB}$, and Groups $\mathrm{A} / \mathrm{B}$ and $\mathrm{A} / \mathrm{AB} / \mathrm{B}$ each differed from Group $A / A B$. On Block 8 , Group Cond differed from Groups $\mathrm{A} / \mathrm{AB} / \mathrm{B}$ and $\mathrm{A} / \mathrm{AB}$, and Groups $\mathrm{A} / \mathrm{B}$ and $\mathrm{A} / \mathrm{AB} / \mathrm{B}$ differed from Group $\mathrm{A} / \mathrm{AB}$ (all $p s<.05$ ). No other pairwise comparisons were significant.

To ensure that use of the elevation ratio was appropriate, the mean number of responses in the pre-CS periods for each five-trial block (i.e., background response rate) was subjected to a two-factor ANOVA (group $\times$ block). This analysis revealed no statistically significant difference between the groups $(F<1)$ and neither was the group $\times$ session interaction significant $(p>.05)$. Thus, there were no statistically significant differences in the background rate of responding in this experiment. The mean rates of responding in the pre-CS periods, collapsed over all five-trial blocks, were 2.69 for Group A/B, 2.17 for Group A/AB, 2.15 for Group A/AB/B, and 1.46 for Group Cond.

These data provide confirmation that a latent inhibition effect can be generated by using the present procedure (see also Reed, 1995a, 1995b). These results also corroborate the finding that exposure to a blocking-oflatent-inhibition design results in retarded acquisition of conditioned responding relative to a condition in which exposure was given to the nontarget stimulus and then the target stimulus alone (see also Reed, 1995a, 1995b). Exposure to the target stimulus alone, following exposure to the compound stimulus, resulted in greater levels of conditioning to the target than in the group exposed only to the blocking-of-latent-inhibition treatment. This result is paradoxical, given that the former group received more preexposure to the target stimulus than did the group receiving the blocking-of-latent-inhibition treatment.

\section{EXPERIMENT 2}

In the second experiment, we attempted to confirm the previous results and extend the investigation of postcompound manipulations in order to test further the associative interference account of these manipulations. In addition to presentation of the target cue following the compound, it is possible, of course, to present the nontarget stimulus alone following compound exposure. According to an associative interference account, such a procedure would produce the pattern of results opposite to that observed when the target $(B)$ is presented following the compound. In the latter case, the $\mathrm{B}-\mathrm{A}$ association is extinguished and, hence, the indirect B-no-event association is lost. However, if it is assumed that the associations formed during compound exposure are directional, then presentation of $A$ alone would not extinguish the $\mathrm{B}-\mathrm{A}$ association (it should be noted that there is evidence that such directional associations do not occur in flavor-aversion paradigms; cf. Speers et al., 1980). Rather, additional presentations of stimulus A would tend, if anything, to strengthen the A-no-event association and, hence, to strengthen the indirect $\mathrm{B}$-no-event association. Thus, this procedure should result in a level of latent inhibition at least as great as that observed in the group given the blocking-of-latent-inhibition treatment.

\section{Method}

Subjects and Apparatus. Forty-eight experimentally naive, male, Sprague-Dawley rats served in the present experiment. All the rats were 5-6 months old at the start of the study, had a free-feeding body-weight range of $395-600 \mathrm{~g}$, and were maintained as in Experiment 1. The apparatus was identical to that used in Experiment 1.

Procedure. The experiment was conducted in two replications. In each replication there were 24 animals, and these subjects were divided into three equal groups $(n=8)$. In Phase 1 , all subjects received twelve 30 -min sessions in which they were presented with ten 30-sec presentations of the nontarget stimulus (the overhead light). In Phase 2, all groups received four 30-min sessions in which they were presented with ten 30 -sec compound presentations of the overhead light and traylight (the target).

In Phase 3, Group A/AB/B received four 30-min sessions in which they were presented with ten 30 -sec presentations of the traylight (the target). Group $\mathrm{A} / \mathrm{AB} / \mathrm{A}$ received four 30 -min sessions in which they were presented with ten 30 -sec presentations of the overhead light. Subjects in Group A/AB were placed in the chamber for four 30-min sessions but received no programmed stimuli presentations.

In the final, conditioning phase, all groups received four 30-min sessions in which 30 -sec presentations of the traylight were followed by the presentation of one food pellet. All ISIs in the present experiment were $2.5 \mathrm{~min}$.

\section{Results and Discussion}

The mean elevation ratios for each five-trial block were subjected to a three-factor ANOVA (group $\times$ block $X$ replication). This revealed that neither the main effect 
of replication nor any of the interactions involving this term were significant (all $p \mathrm{~s}>.15$ ). Figure 2 displays the group mean elevation ratios averaged over each fivetrial block of the conditioning phase collapsed over both replications.

Inspection of these data reveals that Group $\mathrm{A} / \mathrm{AB} / \mathrm{B}$ generally displayed elevation ratios that were higher than those of the other two groups. Group A/AB displayed a level of conditioned responding higher than that of Group $\mathrm{A} / \mathrm{AB} / \mathrm{A}$. The ANOVA revealed a significant main effect of group $[F(2,42)=12.67, p<.01]$, a significant main effect of block $[F(7,294)=12.09, p<$ $.01]$, and a significant interaction between these factors $[F(14,294)=3.26, p<.01]$. To analyze these data further, the simple effect of group at each block was analyzed. These tests revealed a statistically significant difference between the groups on Blocks 3-8, inclusive [smallest $F(2,261)=4.80, p<.01$ ]. Newman-Keuls tests were conducted on the blocks for which there was a significant simple effect of group. On Blocks 3 and 8 , Groups $A / A B / B$ and $A / A B$ differed from Group $A / A B$. On Block 4, Group $A / A B / B$ differed from both Group $\mathrm{A} / \mathrm{AB}$ and Group $\mathrm{A} / \mathrm{AB} / \mathrm{A}$. On Blocks 5-7, all groups differed from each other. No other pairwise comparisons were significant.

The mean number of responses in the pre-CS periods for each five-trial block was subjected to a three-factor ANOVA (group $\times$ block $\times$ replication) that revealed only a significant replication $\times$ block interaction $[F(7,294)=$ $5.09, p<.01]$. No other effect was significant. The mean rates of responding in each pre-CS period collapsed over all five-trial blocks was: 0.98 for Group $\mathrm{A} / \mathrm{AB}, 1.59$ for Group $\mathrm{A} / \mathrm{AB} / \mathrm{A}$, and 1.57 for Group $\mathrm{A} / \mathrm{AB} / \mathrm{B}$. This indi-

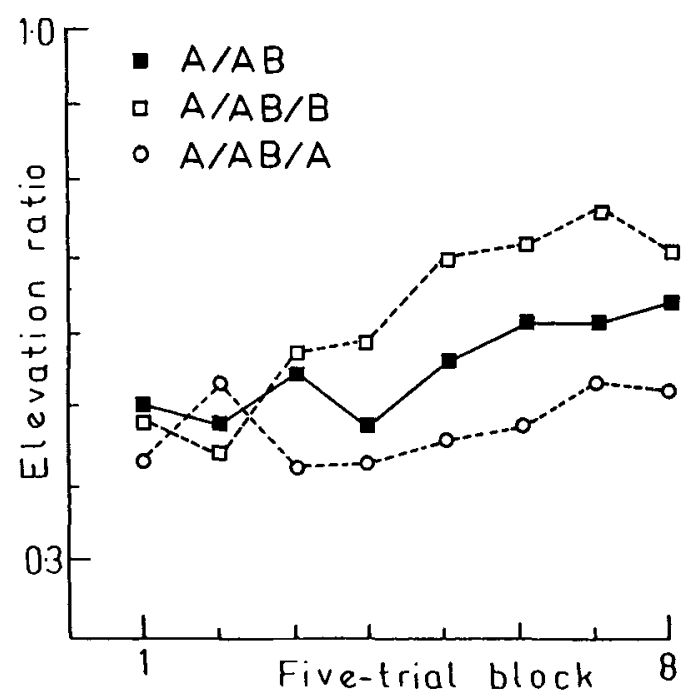

Figure 2. Results from Experiment 2. Group-mean elevation ratios for each five-trial block of conditioning with stimulus $B$. Group $A / A B=$ exposure to nontarget (A) before target/nontarget compound (AB). Group $\mathbf{A} / \mathbf{A B} / \mathbf{B}=$ exposure to nontarget $(A)$, then target/nontarget compound (AB), then exposure to the target (B). Group $A / A B / A=$ exposure to nontarget (A), then to target/nontarget compound (AB), then to nontarget (A). cates that the differences in elevation ratios are not attributable to differences in baseline rates of responding.

As in the previous experiment, subjects given exposure to the target stimulus after the blocking-of-latentinhibition treatment showed relatively good conditioning compared with a group that received only the blocking-of-latent-inhibition training. The novel finding from this experiment is that subjects given exposure to the nontarget stimulus after exposure to the compound displayed worse conditioning than did the subjects in the blocking-of-latent-inhibition group. This finding can be accommodated within the associative interference view if it is assumed that during the third phase of preexposure, when stimulus A was presented alone, this stimulus continued to be associated with no event. In turn, this would strengthen the second-order association between $\mathrm{B}$ and no event which is putatively mediated by the B-A association.

\section{EXPERIMENT 3}

In the third experiment, we explored an alternative to the associative interference explanation of the "extinction" of the latent-inhibition effect. According to Hall and Pearce (1982), the associability of a preexposed stimulus may be restored (i.e., conditioning will proceed more rapidly) if, following the initial preexposure phase, that stimulus is presented along with an unexpected event. In the "extinction-of-latent-inhibition" treatment (A/AB/B), presentation of the target stimulus alone in the final phase of preexposure might constitute an unexpected event. The subjects' previous exposure to the target was in the presence of the nontarget stimulus, the omission of which might be considered surprising. This surprising event might restore the associability of the target stimulus (see Siddle, Booth, \& Packer, 1987).

In Experiment 3, we attempted to explore the account of the "extinction-of-latent-inhibition" effect on the basis of restoration of associability. To this end, three groups of rats were trained. Two groups were similar to those described above in the "extinction-of-latent-inhibition" experiments (i.e., $\mathrm{A} / \mathrm{AB}$ and $\mathrm{A} / \mathrm{AB} / \mathrm{B}$ ). The third group received the same stimulus presentations as Group $\mathrm{A} / \mathrm{AB} / \mathrm{B}$, but had the order of the last two phases reversed, so that presentations of the target stimulus (B) alone occurred prior to presentations of the target/nontarget compound (i.e., $\mathrm{A} / \mathrm{B} / \mathrm{AB}$ ). For the latter group, the presentation of target stimulus in the last phase of exposure can be considered surprising, since this occurs in the presence of the nontarget stimulus (A), whereas, previously, the target had been presented in the absence of the nontarget stimulus. If the associability-based explanation is correct, both Group $\mathrm{A} / \mathrm{AB} / \mathrm{B}$ and Group $\mathrm{A} / \mathrm{B} /$ $A B$ should show better conditioning than the $A / A B$ group. In contrast, should the associative interference account prove to be correct, only the group receiving the "extinction-of-latent-inhibition" training (i.e., A/AB/B) should show good conditioning as compared with that of the enhanced latent inhibition group ( $\mathrm{A} / \mathrm{AB})$. 


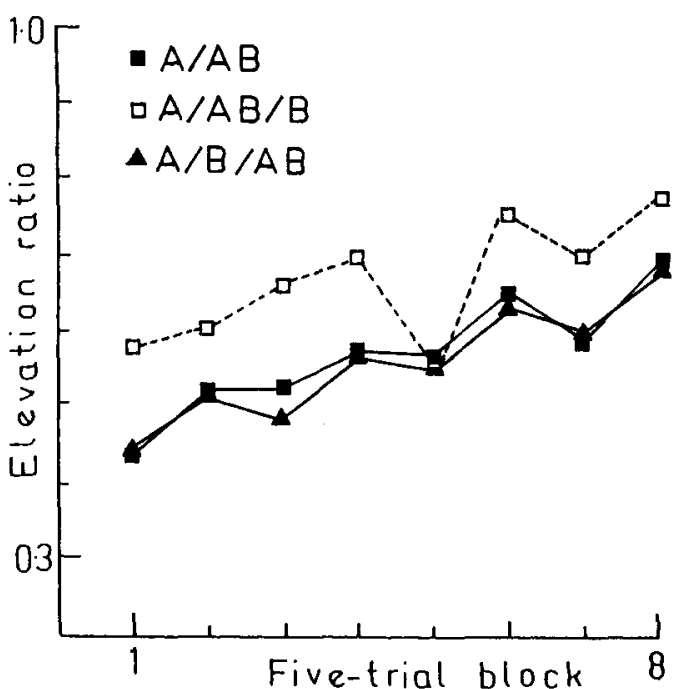

Figure 3. Results from Experiment 3. Group-mean elevation ratios for each five-trial block of conditioning with stimulus $B$. Group $A / A B=$ exposure to nontarget (A) before target/nontarget compound $(A B)$. Group $A / A B / B=$ exposure to nontarget $(A)$, then target/nontarget compound $(A B)$, then exposure to the target (B). Group $A / B / A B=$ exposure to nontarget $(A)$, then to the target $(B)$, then to target/nontarget compound $(A B)$.

\section{Method}

Subjects and Apparatus. Forty-eight experimentally naive, male, Sprague-Dawley rats were used in two replications. The subjects were all 5-6 months old at the start of the experiment, had a free-feeding body weight range of $345-600 \mathrm{~g}$, and were maintained as in Experiment 1. The apparatus was that used in Experiment 1.

Procedure. For each of the replications, the subjects were divided into three equal groups $(n=8)$. In Phase 1 , all subjects received twelve 30 -min sessions in which they were presented with ten 30 -sec presentations of the nontarget stimulus (the overhead light). In Phase 2, Groups $\mathrm{A} / \mathrm{AB}$ and $\mathrm{A} / \mathrm{AB} / \mathrm{B}$ received four 30 -min sessions in which they were presented with ten $30-\mathrm{sec}$ compound presentations of the overhead light and traylight (the target). Group $\mathrm{A} / \mathrm{B} / \mathrm{AB}$ received four 30 -min sessions in which they were presented with ten $30-\mathrm{sec}$ presentations of the traylight (the target).

In Phase 3, Group $\mathrm{A} / \mathrm{AB} / \mathrm{B}$ received four 30 -min sessions in which they were presented with ten 30 -sec presentations of the traylight (the target). Group $\mathrm{A} / \mathrm{B} / \mathrm{AB}$ received four 30 -min sessions in which they were presented with ten $30-\mathrm{sec}$ presentations of a compound stimulus comprising the traylight and the overhead light. Group A/AB were placed in the chamber for four, $30-\mathrm{min}$ sessions but received no programmed stimuli presentations.

In the final, conditioning phase, all groups received four 30 -min sessions in which $30-$ sec presentations of the traylight were followed by the presentation of one food pellet. All ISIs in the present experiment were $2.5 \mathrm{~min}$.

\section{Results and Discussion}

The data were subjected to a three-factor ANOVA (group $X$ five-trial block $\times$ replication). This revealed neither a main effect of replication nor any significant interaction involving this term (all $F \mathrm{~s}<1$ ).

Figure 3 displays the group mean elevation ratios averaged over each five-trial block of the conditioning phase collapsed across replication. Group A/AB/B generally displayed a higher elevation ratio than either of the other two groups. Groups $\mathrm{A} / \mathrm{AB}$ and $\mathrm{A} / \mathrm{B} / \mathrm{AB}$ displayed similar levels of conditioned responding. The ANOVA revealed a significant main effect of group $[F(2,42)=$ $7.02, p<.01]$ and a significant main effect of block $[F(7,294)=10.52, p<.01]$ but no significant interaction between these factors $(F<1)$. To analyze these data further, the group mean elevation ratios over all eight blocks were taken; these scores were 0.68 for Group $\mathrm{A} / \mathrm{AB} / \mathrm{B}$, 0.57 for Group $\mathrm{A} / \mathrm{B} / \mathrm{AB}$, and 0.57 for Group $\mathrm{A} / \mathrm{AB}$. Newman-Keuls tests revealed that Group A/AB/B differed significantly from each of the other two groups $(p \mathrm{~s}<.05)$

The mean number of responses in the pre-CS periods for each five-trial block was subjected to a three-factor ANOVA (group $\times$ block $\times$ replication) that revealed no statistically significant differences. The mean rate of responding in each pre-CS period collapsed over all fivetrial blocks was 1.97 for Group A/AB, 2.13 for Group $\mathrm{A} / \mathrm{AB} / \mathrm{B}$, and 2.30 for Group $\mathrm{A} / \mathrm{B} / \mathrm{AB}$.

Subjects given the "extinction-of-latent-inhibition" treatment showed relatively good conditioning compared with a group that received only the $\mathrm{A} / \mathrm{AB}$ preexposure. However, this attenuation of latent inhibition was not noted in a group which received the same amount of exposure to the target and nontarget stimuli, but which received exposure to the compound stimulus following exposure to the target alone (i.e., Group $\mathrm{A} / \mathrm{B} / \mathrm{AB}$ ). If better conditioning in Group $\mathrm{A} / \mathrm{AB} / \mathrm{B}$ was the result of the unexpected presentation of $\mathrm{B}$ alone following presentation of the $A B$ compound, then a similar beneficial effect on conditioning might have been expected in Group $\mathrm{A} / \mathrm{B} / \mathrm{AB}$, in which there was an unexpected presentation of the nontarget (A) in compound with the target (B), after exposure to the target (B) alone. These results, therefore, run counter to those expected on the basis of a restoration-of-associability account of the "extinctionof-latent-inhibition" effect.

\section{EXPERIMENT 4}

In the preceding experiment it was assumed that the removal of stimulus $\mathrm{A}$ in Group $\mathrm{A} / \mathrm{AB} / \mathrm{B}$ would be as surprising as the addition of this stimulus in Group $\mathrm{A} / \mathrm{B} / \mathrm{AB}$. However, this may not be the case. It is conceivable that the addition of stimulus $A$ in the third phase of preexposure would not be as surprising as its removal, stimulus A having already been an object of habituation in the first phase of exposure. There is some evidence to suggest that an orienting response (a measure of associability) is not restored during a habituation procedure when presentation is shifted between contexts, if the context into which the subjects are shifted is familiar or habituated (see Hall \& Channel, 1985). If this were the case in the present procedure, then the results of the above experiment would be predictable by an associability account.

In Experiment 4, we attempted to restore the associability of the target stimulus in the final phase of nonreinforced preexposure by presenting a novel, nonhabitu- 
ated stimulus along with that target. Such a procedure has previously been found to restore associability in other paradigms (Hall \& Pearce, 1982). If this manipulation were to prove more successful in restoring the associability of the target stimulus, then conditioning should proceed as rapidly for this group as it does for the group receiving the "extinction-of-latent-inhibition" training. However, only in the latter group is a source of noevent learning lost, and the associative interference account therefore predicts that this "extinction-of-latent-inhibition" group will show better conditioning than the former group.

\section{Method}

Subjects and Apparatus. Twenty-four experimentally naive, male Sprague-Dawley rats served in the present experiment. All the rats were 4-5 months old at the start of the study, had a free-feeding body weight range of $300-380 \mathrm{~g}$, and were maintained as in Experiment 1 . The apparatus was identical to that used in Experiment 1 .

Procedure. The subjects were divided into three equal groups. In Phase 1, all subjects received twelve 30 -min sessions in which they were presented with ten 30 -sec presentations of the nontarget stimulus (for half the animals in each group, this was the overhead light; for the other half, this was the center light). In Phase 2, Group $\mathrm{A} / \mathrm{AB} / \mathrm{B}$ received four $30-\mathrm{min}$ sessions in which they were presented with ten 30 -sec compound presentations of the overhead light (or the center light) and traylight (the target). Groups $A / B / A B$ and $A / B / C B$ received four 30 -min sessions in which they were given ten 30-sec presentations of the traylight (the target).

In Phase 3, Group A/AB/B received four 30-min sessions in which they were given ten $30-\mathrm{sec}$ presentations of the traylight (the target). Group $A / B / A B$ received four 30-min sessions in which they were given ten $30-\mathrm{sec}$ presentations of a compound stimulus comprising the traylight and either the overhead light or center light (i.e., the same stimulus presented in Phase 1). Group A/B/CB received four 30 -min sessions in which they were given ten 30 -sec presentations of a compound stimulus comprising the traylight and the overhead light or center light (i.e., the stimulus not presented in Phase 1).

In the final, conditioning phase, all groups received four $30-\mathrm{min}$ sessions in which 30-sec presentations of the traylight were followed by the presentation of one food pellet. All ISIs in the present experiment were $2.5 \mathrm{~min}$.

\section{Results and Discussion}

The group mean elevation ratios, averaged over each five-trial block of the conditioning phase and displayed in Figure 4, reveal that Group A/AB/B generally displayed a higher elevation ratio than did either of the other two groups. For Groups $\mathrm{A} / \mathrm{B} / \mathrm{AB}$ and $\mathrm{A} / \mathrm{B} / \mathrm{CB}$, the levels of conditioned responding were similar to each other. A two-factor ANOVA (group $\times$ block) revealed no main effect of group $(F<1)$ but did reveal a significant main effect of block $[F(7,147)=7.04, p<.001]$ and a significant interaction between the factors $[F(14,147)=2.00$, $p<.05]$. Simple main effect analyses revealed a difference between groups on Block $8[F(2,104)=4.13$, $p<.05]$. Newman-Keuls tests conducted on this block revealed that Group A/AB/B differed from each of the other two groups. No other pairwise differences were significant.

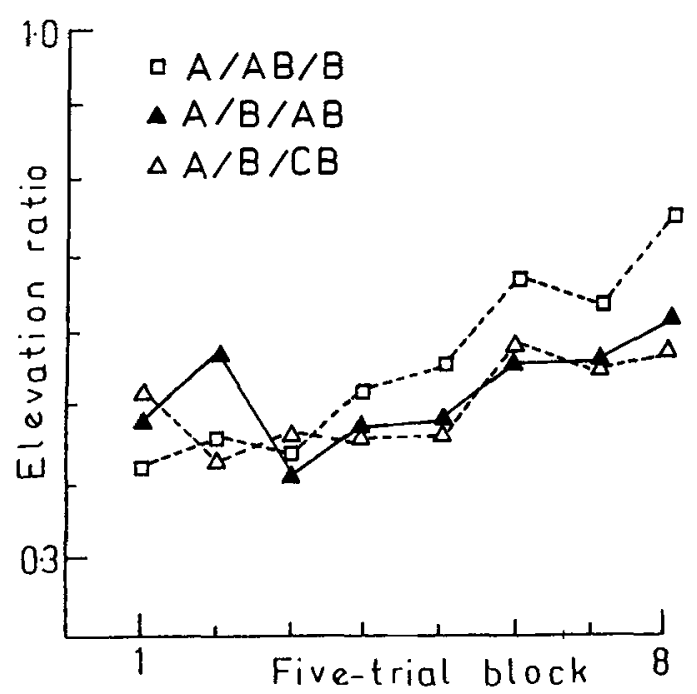

Figure 4. Results from Experiment 4. Group-mean elevation ratios for each five-trial block of conditioning with stimulus $B$. Group $A / A B / B=$ exposure to nontarget (A), then target/nontarget compound $(\mathrm{AB})$, then exposure to the target $(B)$. Group $\mathrm{A} / \mathrm{B} / \mathrm{AB}=$ exposure to nontarget $(\mathrm{A})$, then exposure to the target (B), then target/nontarget compound (AB). Group $A / B / C B=e x-$ posure to nontarget $(A)$, then to the target $(B)$, then to target/nontarget compound (CB).

The mean number of responses in the pre-CS periods for each five-trial block was subjected to a two-factor ANOVA (group $\times$ session) that revealed no statistically significant difference between the groups or an interaction $\left(F_{\mathrm{S}}<1\right)$. There was a significant main effect of block, but this did not reflect any systematic trend in the data. The mean rate of responding in each pre-CS period collapsed over all five-trial blocks was 2.41 for Group $\mathrm{A} / \mathrm{B} / \mathrm{AB}, 2.82$ for Group $\mathrm{A} / \mathrm{B} / \mathrm{CB}$, and 2.63 for Group $\mathrm{A} / \mathrm{AB} / \mathrm{B}$.

These results confirm that subjects given an "extinctionof-latent-inhibition" treatment (i.e., A/AB/B) showed relatively good conditioning as compared with a group that received exposure to the target stimulus prior to exposure to the compound (i.e., A/B/AB). A group that received a novel stimulus along with the target stimulus in the final phase of exposure $(\mathrm{A} / \mathrm{B} / \mathrm{CB})$ demonstrated similarly retarded acquisition of conditioned responding to the latter group. If the better conditioning in Group $\mathrm{A} / \mathrm{AB} / \mathrm{B}$ than in Group $A / B / A B$ was the result of the removal of $A$ in the former group being more surprising than the addition of A (an already habituated stimulus) in the latter group, then a similar beneficial effect on conditioning might have been expected in Group $\mathrm{A} / \mathrm{B} / \mathrm{CB}$. In the latter group there was an unexpected presentation of a novel stimulus (C) in compound with the target (B), after initial exposure to the target (B) alone (see Hall \& Pearce, 1982). That this manipulation failed to produce faster conditioning than in Group $\mathrm{A} / \mathrm{B} / \mathrm{AB}$ suggests that the explanation for the "extinction-of-latent-inhibition" effect in Group $\mathrm{A} / \mathrm{AB} / \mathrm{B}$ in terms of restoration of associability can- 
not accommodate these data. Rather, the results remain consistent with an associative interference explanation. Only in Group $\mathrm{A} / \mathrm{AB} / \mathrm{B}$ would a source of an association between $B$ and no event be lost (i.e., that produced by second-order conditioning between $B$ and $A$ ). In the other two groups, conditioning to $B$ might be expected to be poor since, in both cases, the subjects would acquire direct B-no-event associations and also second-order no-event associations through the compound conditioning phase.

\section{GENERAL DISCUSSION}

The present experiments demonstrated enhanced levels of latent inhibition following exposure to a blockingof-latent-inhibition procedure as opposed to exposure to the target and nontarget stimuli in isolation. Furthermore, exposure to the target stimulus in isolation following exposure to the compound stimulus attenuated this effect: conditioning proceeded relatively rapidly in a group that received the above treatment as compared with a group that received the blocking-of-latent-inhibition treatment without subsequent nonreinforced exposure to the target.

The results of the present experiments support the view that associative interference has a role in generating latent inhibition (see Hall et al., 1985; Reed, 1995a, $1995 \mathrm{~b}$ ). As outlined in the general introduction, the associative interference account assumes that during nonreinforced exposure to a potential CS, a CS-no-event association is acquired that subsequently retards acquisition of the CS-US association during conditioning. During a blocking-of-latent-inhibition procedure, a subject is taken to acquire a nontarget-no-event association in the first phase of exposure and both a target-no-event and a target-nontarget association in the compoundexposure phase of the procedure. The acquisition of a target-nontarget association allows for second-order conditioning of a target-no-event association through the target-nontarget and the previously established nontarget-no-event associations. The summation of the first- and second-order associations of the target with no event leads to levels of latent inhibition that are enhanced relative to those of a group that does not receive this training. In the extinction-of-latent inhibition procedure, nonreinforced exposure to the target stimulus alone, following the compound exposure, produces extinction of the target-nontarget association and the loss of the second-order association between the target and no event. Thus, levels of interference between the targetno-event and target-event associations are reduced in this group, and conditioning proceeds more rapidly than when this target-alone exposure is not given to the subject following the compound exposure of a blocking-oflatent-inhibition design.

The reduction of latent inhibition noted with the extinction-of-latent inhibition procedure renders unlikely an explanation of these effects based on greater numbers of light presentations per se. Such an account might assume that the nontarget and target stimuli generalize one to the other, and consequently, in the blockingof-latent-inhibition design, the group receiving the compound exposure (i.e., $\mathrm{A} / \mathrm{AB}$ ) has twice as many stimulus exposures in the second phase of the study than does the group not receiving this treatment (i.e., A/B). In the present experiments, the "extinction" group received more light presentations than did the "blocking" group, but, contrary to the above line of reasoning, demonstrated better levels of conditioned responding. The generalization explanation was also addressed by experiments conducted by Reed (1995b) and found wanting. In this series of experiments, the treatment resulting in enhanced latent inhibition (i.e., A/AB) was compared with that of a group receiving both target (B) and nontarget (A) presentations in Phase 2 of the preexposure but presented randomly with respect to each other. Latent inhibition was still significantly greater in the group given compound exposure than in the group presented with the elements of the compound separately. It should be noted that it is not claimed that this manipulation exerted no influence on the results (see Reed, Anderson, Bucknell, \& Foster, 1996).

A number of findings, however, suggest that associative interference is not the only factor involved in generating latent inhibition. The finding that stimuli from the same modality, when exposed in nonreinforced compound prior to conditioning, exert a greater detrimental effect on subsequent latent inhibition than do stimuli from different modalities suggests that there is a perceptual influence on latent inhibition (see Honey \& Hall, 1989). However, Reed et al. (1996) have demonstrated that the present enhanced latent inhibition effect can also be generated using stimuli drawn from different modalities (a light and a tone). Thus, the enhanced latent inhibition effect seems not to depend on perceptual processing to the same extent apparent in the "overshadowing" of latent inhibition designs. Finally, it should be mentioned that the effects of preexposure on the elements of a to-beconditioned compound stimulus do not provide unequivocal support for an associative interference account of latent inhibition (see Baker, Haskins, \& Hall, 1990). In sum, the available evidence suggests that a combination of factors is responsible for latent inhibition, and that it would be premature to conclude that associative interference was not among these factors.

\section{REFERENCES}

Baker, A. G., Haskins, C. E., \& Hall, G. (1990). Stimulus generalization decrement in latent inhibition to a compound following exposure to the elements or the compound. Animal Learning \& Behavior, 18, 162-170.

HaLl, G., \& Channel, S. (1985). Differential effects of context change on latent inhibition and on the habituation of an orienting response. Journal of Experimenial Psychology: Animal Behavior Processes, 11. 470-481.

HALL, G., \& HONEY, R. C. (1989). Perceptual and associative learning. 
In S. B. Klein \& R. R. Mowrer (Eds.), Contemporary learning theories (pp. 117-147). Hillsdale, NJ: Erlbaum.

Hall, G., KaYe, H., \& PeArCE, J. M. (1985). Attention and conditioned inhibition. In R. R. Miller \& N. E. Spear (Eds.), Information processing in animals: Conditioned inhibition (pp. 185-207). Hillsdale, $\mathrm{NJ}$ : Erlbaum.

HaLl, G., \& Pearce, J. M. (1982). Restoring the associability of a preexposed CS by a surprising event. Qtarterly Journal of Experimental Psychology, 34B, 127-140.

Holland, P. C. (1980). Second-order conditioning with and without unconditioned stimulus presentations. Journal of Experimental Psychology: Animal Behavior Processes, 6, 238-250.

HoNEY, R. C., \& HALL, G. (1988). Overshadowing and blocking procedures in latent inhibition. Quarterly Journal of Experimental $P_{s y-}$ chology, 40B, 163-186.

Honey, R. C., \& HALL, G. (1989). Attenuation of latent inhibition after compound preexposure: Associative and perceptual explanations. Quarterly Journal of Experimental Psychology, 418, 355-368.

REED, P. (1995a). Compound stimuius preexposure effects in an appetitive conditioning procedure. Learning \& Motivation, 26, 1-10.

REED, P. (1995b). Enhanced latent inhibition following compound preexposure. Quarterly Journal of Experimental Psychology, 48B, 32-45.

Reed, P., Anderson, E., Bucknell, V., \& Foster, C. (1996). The in- fluence of type of stimulus on the enhanced latent inhibition effect. Manuscript submitted for publication.

Rescorla, R. A., \& Colwill, R. M. (1983). Within-compound associations in unblocking. Journal of Experimental Psychology: Animal Behavior Processes, 9, 390-400.

Rudy, J. W., Krauter, E. E., \& Gaffuri, A. (1976). Attenuation of the latent inhibition effect by prior exposure to another stimulus. Journal of Experimental Psychology: Animal Behavior Processes, 2, 235-247.

SidDLE, D. A., BOOTH, M. L., \& PACKER, J. S. (1987). Effects of stimulus preexposure on omission responding and omission-produced dishabituation of the human electrodermal response. Quarterly Journal of Experimental Psychology, 39B, 339-363.

SPEers, M, A., Gillan, D. J., \& Rescorla, R. A. (1980). Within compound associations in a variety of compound conditioning procedures. Learning \& Motivation, 11, 135-149.

WAGNER, A. R. (1981). SOP: A model of automatic memory processing in animal behavior. In N. E. Spear \& R. R. Miller (Eds.), Information processing in animals: Memory mechanisms (pp. 5-47). Hills dale, NJ: Erlbaum.

(Manuscript received May 9, 1996;

revision accepted for publication December 8, 1996.) 